\title{
Modulation analysis and optical solitons of perturbed nonlinear Schrödinger equation
}

\author{
A. Houwe ${ }^{a}$, J. Sabi'u $^{b}$, G. Betchewe ${ }^{a}$, M. Inc ${ }^{c, d}$, and S. Y. Doka ${ }^{e}$ \\ ${ }^{a}$ Department of Physics, Faculty of Science, the University of Maroua, P.O Box 814, Maroua, Cameroon. \\ ${ }^{b}$ Department of Mathematics, Faculty of sciences, Northwest University Kano, P.O.Box 3220, Kano, Nigeria. \\ ${ }^{c}$ Firat University, Science Faculty,Department of Mathematics, 23119 Elazig/Turkey. \\ e-mail:minc@firat.edu.tr \\ ${ }^{d}$ Department of Medical Research, China Medical University Hospital, China Medical University, Taichung, Taiwan. \\ e Department of Physics, Faculty of Science, the University of Ngaoundere, P.O Box 454, Cameroon.
}

Received 30 January 2021; accepted 18 February 2021

\begin{abstract}
We investigate modulation analysis and optical solitons of perturbed nonlinear Schrödinger equation (PNLSE). The PNLSE has terms of cubic nonlinearity and self-steepening and spatio-temporal dispersion (STD). Proposed model has been studied by [14, 15] without selfsteepening term. The presence of the STD can help to compensate the low GVD to the model. Bright and dark solitary waves, trigonometric, periodic and singular optical solitons are obtained by some expansion methods including exponential and sinh-Gordon. Obtained results will hold a significant place in the field of nonlinear optical fibers, where solitons are used to codify data.
\end{abstract}

Keywords: Optical soliton; perturbed nonlinear Schrödinger equation; instability modulation.

PACS: $04.20 . J b ; 05.45 . Y v ; 94.05 . F g$.

DOI: https://doi.org/10.31349/RevMexFis.67.040705

\section{Introduction}

Research on exact solutions of nonlinear systems has reached cruising speed these days. The best known solitons solutions have found their applications in several research areas including optical fibers, plasm, biology, and quantum physics, just to name a few. Thus, optical solitons did not remain anonymous for a long time because of their direct implications in trans-continental and trans-oceanic data transport [1-10]. It is important to remember that thanks to the advent of optical solitons, the field of communication has experienced an expansion through high speed data transfer, and guaranteed protection. Thereafter, many theoretical and experimental results have been followed with the mathematical tools to handle them. In this way, exact optical solitons resulting from the nonlinear Schrödinger equations with different nonlinearities have been reported [10-13]. Some of the analytical methods that facilitated the success of these results are expansion methods including modified $\exp (-\psi(\xi))$ function, $\left(G^{\prime} / G\right)$, trial-error, auxiliary equation, rational function, Riccati-Bernoulli sub-ODE , the sine-Gordon expansion, $\exp (-\phi(\xi))$-expansion, see [2-23].

The present work, aims to unearth exact optical solitons to PNLSE $[14,15]$ :

$$
i \psi_{t}+a_{1} \psi_{x t}+a_{2} \psi_{x x}+a_{3}|\psi|^{2} \psi-i \beta\left(|\psi|^{2} \psi\right)_{x}=0,
$$

$\psi(x, t)$ is complex wave profile, $t$ represents temporal variable and $x$ is spatial variable. $a_{1}$ is the STD, $a_{2}$ the coefficient of group velocity dispersion (GVD), while $a_{3}$ is the cubic nonlinearity which will be combined with the dispersion terms in order to build optical solitons. However, $\beta$ is the self-steepening coefficient.

Section 2 concern the traveling-wave solution to transform the PNLSE into to integrable form of nonlinear ordinary differential equation. In Secs. 3 and 4, we apply two integration schemes to look optical solitons and we establish also the existence criteria of the obtained results. In Sec. 5 we discussed the modulation instability of of the obtained results. The last part of the paper is devoted to conclusion. To determine optical solitons to Eq. (1), we will use sinh-Gordon and $\exp (-\Phi(\xi)$ )-expansion techniques with the traveling-wave hypothesis.

\subsection{Traveling waves solution}

It is used the following transformation to (1) to build soliton solution

$$
\psi(x, t)=\phi(\xi) \exp [i \theta(x, t)], \quad \xi=x-v t,
$$

where $\phi$ represents the shape of the soliton and depends on the Kerr non-linearity. However the phase $\theta(x, t)$ is given by

$$
\theta(x, t)=-\kappa x+\omega t+\theta_{0},
$$

Inserting (2) and used (3) into (1) it is obtained the speed of the soliton from the imaginary part

$$
v=\frac{a_{1}(2 \kappa-\omega)}{1-a_{1} \kappa}, \quad 1 \neq a_{1} \kappa .
$$

with the constrains relation on the self-steepening parameters.

$$
\beta=0 .
$$


Here, we omit the SPM term to preserve the obtained bright and dark optical solitons against deformation of the waveform, sensitivity deterioration of the receiver and the transmission limits distance cause by the latter in dispersion optic fibers. Recently, it has been demonstrated numerically that a waveform distortion is caused by the SPM in nonlinear Schrödinger equation in the transmission fiber $[15,18]$.

$$
\begin{aligned}
(-\omega & \left.+a_{1} \kappa \omega+a_{2} \kappa^{2}\right) \phi \\
& +\left(a_{1}^{2} \frac{(2 \kappa-\omega)}{1-a_{1} \kappa}+a_{2}\right) \phi_{\xi \xi}+a_{3} \phi^{3}=0,
\end{aligned}
$$

where $\phi_{\xi \xi}=\partial^{2} \phi / \partial \xi^{2}$.

Now, multiplying Eq. (6) by $\phi_{\xi}$ and integrating once gives

$$
\begin{aligned}
& \left(a_{1}^{2} \frac{(2 \kappa-\omega)}{1-a_{1} \kappa}+a_{2}\right) \phi_{\xi}^{2} \\
& -\left(\omega-a_{1} \kappa \omega-a_{2} \kappa^{2}\right) \phi^{2}+\frac{a_{3}}{2} \phi^{4}=0,
\end{aligned}
$$

Then, after separating variables and integrating with zero constant it is obtained

$$
x-v t=\int \frac{d \phi}{\phi \sqrt{\omega-a_{1} \kappa \omega-a_{2} \kappa^{2}-\frac{a_{3}}{2} \phi^{2}}},
$$

Consequently,

$$
\phi(x, t)=\frac{A}{\cosh [B(x-v t)]},
$$

in which

$$
\begin{aligned}
& A= \pm \sqrt{\frac{a_{3}}{2}}, \\
& B= \pm a_{1} \sqrt{\frac{2 \kappa-\omega}{1-a_{1} \kappa}},
\end{aligned}
$$

and the corresponding chirp-free bright soliton

$$
\begin{aligned}
\psi_{1}(x, t) & =\frac{A}{\cosh [B(x-v t)]} \\
& \times \exp \left[i\left(-\kappa x+\omega t+\theta_{0}\right)\right],
\end{aligned}
$$

Then, the constraint relation from the obtained Eq. (10) and Eq. (11) implies

$$
\left(1-a_{1} \kappa\right)(2 \kappa-\omega)>0
$$

and

$$
a_{3}>0
$$

We note that, in the absence of self-pentification $(\beta=0)$, the asymmetry in the form of a pulse is also absent, which implicitly recognizes the formation of shock waves. Here this absence could also affect the pulse width of the solitons obtained. However, the presence of GVD and cubic nonlinearity reinforces the search for optical solitons which will be at least robust against the formation of shocks. In addition, we also point out that the ODE (7) can be directly integrated, but to obtain specific optical solutions, we choose to do so through two integration techniques.

\section{Sinh-Gordon expansion method}

Following expression is adopted as in [19-21], the detail of the method can be obtained in [19]. By principle of balance from (6) between $\phi_{\xi \xi}$ and $\phi^{3}, N=1$.

$$
\begin{aligned}
\phi(\xi) & =\phi(w(\xi))=A_{0} \\
& +A_{1} \sinh w(\xi)+B_{1} \cosh w(\xi),
\end{aligned}
$$

and $A_{0}, A_{1}$ and $B_{1}$ are parameters to be determined.

To build some special Jacobian elliptic function solution, we used the following expression

$$
\begin{aligned}
\sinh [w(\xi)] & =c s(\xi ; m), \\
\cosh [w(\xi)] & =n s(\xi ; m),
\end{aligned}
$$

$m$ represents modulus of Jacobian functions [19-21].

Now, substituting Eq. (15) along with others derivatives into Eq. (6) and taking into consideration (16a-16b), it is recovered after some computations:

- $\cosh ^{3}(w(\xi))$ :

$$
\begin{aligned}
3 a_{3} A_{1}^{2} B_{1} & +a_{3} B_{1}^{3}-\frac{2 a_{1}^{2} \omega B_{1}}{1-a_{1} \kappa} \\
& +2 a_{2} B_{1}+\frac{4 a_{1}^{2} \kappa B_{1}}{1-a_{1} \kappa}=0,
\end{aligned}
$$

- $\cosh ^{2}(w(\xi))$ :

$$
3 a_{3} A_{0} B_{1}^{2}+3 a_{3} A_{0} A_{1}^{2}=0,
$$

- $\sinh (w(\xi)) \cosh ^{2}(w(\xi))$ :

$$
\begin{aligned}
a_{3} A_{1}^{3} & -\frac{2 a_{1}^{2} \omega A_{1}}{1-a_{1} \kappa}+2 a_{2} A_{1} \\
& +3 a_{3} A_{1} B_{1}^{2}+\frac{4 a_{1}^{2} \kappa A_{1}}{1-a_{1} \kappa}=0
\end{aligned}
$$

- $\cosh (w(\xi))$ :

$$
\begin{aligned}
a_{1} \kappa \omega B_{1} & +a_{2} B_{1} c-\frac{4 a_{1}^{2} \kappa B_{1}}{1-a_{1} \kappa}-3 a_{3} A_{1}^{2} B_{1}-\frac{a_{1}^{2} \omega B_{1} c}{1-a_{1} \kappa} \\
& -\omega B_{1}-2 a_{2} B_{1}+\frac{2 a_{1}^{2} \omega B_{1}}{1-a_{1} \kappa}+3 a_{3} A_{0}^{2} B_{1} \\
& +\frac{2 a_{1}^{2} \kappa B_{1} c}{1-a_{1} \kappa}+a_{2} \kappa^{2} B_{1}=0
\end{aligned}
$$


- $\sinh (w(\xi)) \cosh (w(\xi))$ :

$$
6 a_{3} A_{0} A_{1} B_{1}=0
$$

- Constant:

$$
a_{2} \kappa^{2} A_{0}-3 a_{3} A_{0} A_{1}^{2}+a_{1} \kappa \omega A_{0}+a_{3} A_{0}^{3}-\omega A_{0}=0
$$

- $\sinh (w(\xi))$ :

$$
-\omega A_{1}-a_{3} A_{1}^{3}+a_{1} \kappa \omega A_{1}+\frac{2 a_{1}^{2} \kappa A_{1} c}{1-a_{1} \kappa}+\frac{a_{1}^{2} \omega A_{1}}{1-a_{1} \kappa}-\frac{a_{1}^{2} \omega A_{1} c}{1-a_{1} \kappa}-\frac{2 a_{1}^{2} \kappa A_{1}}{1-a_{1} \kappa}+3 a_{3} A_{0}^{2} A_{1}-a_{2} A_{1}+a_{2} A_{1} c+a_{2} \kappa^{2} A_{1}=0,
$$

With help of Maple 14, we solve Eqs. (17-23), and it is recovered the following solution families

- Family 1:

$$
\begin{aligned}
& A_{0}=0, A_{1}=0, B_{1}=B_{1}, \\
& \omega=-\frac{-\kappa^{2} a_{3} B_{1}^{2}+2 a_{3} B_{1}^{2}-c a_{3} B_{1}^{2}-4 a_{1}^{2} \kappa^{3}+a_{1} \kappa^{3} a_{3} B_{1}^{2}-2 a_{3} B_{1}^{2} a_{1} \kappa+a_{1} c a_{3} B_{1}^{2} \kappa}{2\left(2 a_{1} \kappa-1\right)} .
\end{aligned}
$$

\section{- Family 2:}

$$
\begin{aligned}
A_{0} & =0, A_{1}=A_{1}, B_{1}=0, \\
\omega & =-\frac{-\kappa^{2} a_{3} A_{1}^{2}-a_{3} A_{1}^{2}-c a_{3} A_{1}^{2}-4 a_{1}^{2} \kappa^{3}+a_{1} \kappa^{3} a_{3} A_{1}^{2}+a_{3} A_{1}^{2} a_{1} \kappa+a_{1} c a_{3} A_{1}^{2} \kappa}{2\left(2 a_{1} \kappa-1\right)} .
\end{aligned}
$$

\section{- Family 3:}

$$
\begin{aligned}
& A_{0}=0, \quad A_{1}=B_{1}, \quad B_{1}=B_{1}, \\
& \omega=-\frac{-2 \kappa^{2} a_{3} B_{1}^{2}+a_{3} B_{1}^{2}-2 c a_{3} B_{1}^{2}-2 a_{1}^{2} \kappa^{3}-a_{3} B_{1}^{2} a_{1} \kappa+2 a_{1} \kappa^{3} a_{3} B_{1}^{2}+2 a_{1} c a_{3} B_{1}^{2} \kappa}{2 a_{1} \kappa-1} .
\end{aligned}
$$

\section{- Family 4:}

$$
\begin{aligned}
A_{0} & =0, \quad A_{1}=-B_{1}, \quad B_{1}=B_{1}, \\
\omega & =-\frac{-2 \kappa^{2} a_{3} B_{1}^{2}+a_{3} B_{1}^{2-} 2 c a_{3} B_{1}^{2}-2 a_{1}^{2} \kappa^{3}-a_{3} B_{1}^{2} a_{1} \kappa+2 a_{1} \kappa^{3} a_{3} B_{1}^{2}+2 a_{1} c a_{3} B_{1}^{2} \kappa}{2 a_{1} \kappa-1} .
\end{aligned}
$$

\subsection{Jacobian elliptic function solutions}

From family 1 , it is obtained the following Jacobian elliptic function solutions

$$
\psi_{11}(x, t)=B_{1} n s(\xi, m) \times \exp \left(i\left[-\kappa x+\omega t+\theta_{0}\right]\right) .
$$

From family 2, we recovered

$$
\psi_{12}(x, t)=A_{1} c s(\xi, m) \times \exp \left(i\left[-\kappa x+\omega t+\theta_{0}\right]\right) .
$$

From family 3, it is gained the combined Jacobian elliptic function solutions

$$
\psi_{13}(x, t)=B_{1}\{c s(\xi, m)+n s(\xi, m)\} \exp \left(i\left[-\kappa x+\omega t+\theta_{0}\right]\right) .
$$

Then, from family 4 it is acquired the combined Jacobian elliptic function solutions

$$
\psi_{14}(x, t)=B_{1}\{-c s(\xi, m)+n s(\xi, m)\} \exp \left(i\left[-\kappa x+\omega t+\theta_{0}\right]\right) .
$$




\subsection{Trigonometric and singular solutions}

From family 1 , when $m \rightarrow 1$,it is obtained

$$
\psi_{15}(x, t)=B_{1} \operatorname{coth}\left(x-\frac{a_{1}(2 \kappa-\omega)}{1-a_{1} \kappa} t\right) \exp \left(i\left[-\kappa x+\omega t+\theta_{0}\right]\right) .
$$

Then from family 2 , when $m \rightarrow 1$, it is recovered singular optical solutions

$$
\psi_{16}(x, t)=A_{1} \operatorname{csch}\left(x-\frac{a_{1}(2 \kappa-\omega)}{1-a_{1} \kappa} t\right) \exp \left(i\left[-\kappa x+\omega t+\theta_{0}\right]\right) .
$$

To family 3 and 4 , the combined optical solutions is obtained, when $m \rightarrow 1$.

$$
\psi_{17}(x, t)=B_{1}\left(\operatorname{csch}\left[x-\frac{a_{1}(2 \kappa-\omega)}{1-a_{1} \kappa} t\right]+\operatorname{coth}\left[x-\frac{a_{1}(2 \kappa-\omega)}{1-a_{1} \kappa} t\right]\right) \exp \left(i\left[-\kappa x+\omega t+\theta_{0}\right]\right) .
$$

and

$$
\psi_{18}(x, t)=B_{1}\left(-\operatorname{csch}\left[x-\frac{a_{1}(2 \kappa-\omega)}{1-a_{1} \kappa} t\right]+\operatorname{coth}\left[x-\frac{a_{1}(2 \kappa-\omega)}{1-a_{1} \kappa} t\right]\right) \exp \left(i\left[-\kappa x+\omega t+\theta_{0}\right]\right) .
$$

\subsection{Periodic and singular solutions}

When $m \rightarrow 0$, it is recovered periodic and singular solutions to (1) From family 1, it is obtained

$$
\psi_{19}(x, t)=B_{1} \csc \left(x-\frac{a_{1}[2 \kappa-\omega]}{1-a_{1} \kappa} t\right) \exp \left(i\left[-\kappa x+\omega t+\theta_{0}\right]\right) .
$$

From family 2, we recovered

$$
\psi_{20}(x, t)=A_{1} \cot \left(x-\frac{a_{1}[2 \kappa-\omega]}{1-a_{1} \kappa} t\right) \exp \left(i\left[-\kappa x+\omega t+\theta_{0}\right]\right) .
$$

From family 3 , it is gained the combined solutions

$$
\psi_{21}(x, t)=B_{1}\left(\cot \left[x-\frac{a_{1}\{2 \kappa-\omega\}}{1-a_{1} \kappa} t\right]+\csc \left[x-\frac{a_{1}\{2 \kappa-\omega\}}{1-a_{1} \kappa} t\right]\right) \exp \left(i\left[-\kappa x+\omega t+\theta_{0}\right]\right) .
$$

Then, from family 4 it is acquired the combined optical solutions

$$
\psi_{22}(x, t)=B_{1}\left(-\cot \left[x-\frac{a_{1}\{2 \kappa-\omega\}}{1-a_{1} \kappa} t\right]+\csc \left[x-\frac{a_{1}[2 \kappa-\omega]}{1-a_{1} \kappa} t\right]\right) \exp \left(i\left[-\kappa x+\omega t+\theta_{0}\right]\right) .
$$

\section{3. $\exp (-\Phi(\xi))$-expansion method}

Let

$$
\phi(x, t)=\sum_{i=0}^{N} A_{i}(\exp (-\Phi(\xi)))^{i},
$$

in which $A_{i}$ are unknown parameters, while $\Phi(\xi)$ satisfies [22,23] the ODE:

$$
\Phi^{\prime}(\xi)=\exp (-\Phi(\xi))+\mu \exp (\Phi(\xi))+\lambda,
$$

Using homogeneous principle of balance between $\phi_{\xi \xi}$ and $\phi^{3}$ in (6), it is obtained $N=1$. Hence,

$$
\phi(x, t)=A_{0}+A_{1}(\exp (-\Phi(\xi))),
$$

Substituting Eqs. (41) and (42) into (6) leads to a polynomial in $(\exp (-\Phi(\xi)))$. By MAPLE software and letting coefficients of $(\exp (-\Phi(\xi)))$ to 0 :

- Set 1: $A_{0}=A_{0}, A_{1}=A_{1}, \kappa=\frac{1}{a_{1}}, \omega=\frac{2}{a_{1}}$ 
- Set 2:

$$
\begin{aligned}
& A_{0}=\lambda \sqrt{\frac{2 a_{1}{ }^{3} \kappa^{2}-2 a_{1}^{2} \kappa+2 a_{2} a_{1} \kappa-a_{2}}{a_{3}\left(2 a_{1}^{2} \kappa^{2}-a_{1}^{2} \lambda^{2}+4 a_{1}^{2} \mu-4 a_{1} \kappa+2\right)}}, \quad A_{1}=2\left(\sqrt{\frac{2 a_{1}^{3} \kappa^{2}-2 a_{1}^{2} \kappa+2 a_{2} a_{1} \kappa-a_{2}}{a_{3}\left(2 a_{1}^{2} \kappa^{2}-a_{1}^{2} \lambda^{2}+4 a_{1}^{2} \mu-4 a_{1} \kappa+2\right)}}\right), \\
& \omega=\frac{2 a_{2} \kappa^{2}-a_{2} \lambda^{2}+4 a_{2} \mu-2 a_{2} \kappa^{3} a_{1}+a_{2} \lambda^{2} a_{1} \kappa-4 a_{2} \mu a_{1} \kappa-2 a_{1}^{2} \kappa \lambda^{2}+8 a_{1}^{2} \kappa \mu}{2 a_{1}^{2} \kappa^{2}-a_{1}^{2} \lambda^{2}+4 a_{1}^{2} \mu-4 a_{1} \kappa+2}, \quad \kappa=\kappa .
\end{aligned}
$$

From set 1 , it is obtained five families of solutions:

(1.1) If $\lambda^{2}-4 \mu>0$, and $\mu \neq 0$, it is acquired

$$
\psi_{21,1}(x, t)=\left(A_{0}+\frac{2 A_{1} \mu}{-\sqrt{-4 \mu+\lambda^{2}} \tanh \left[\frac{1}{2} \sqrt{-8 \mu+2 \lambda^{2}}\left\{\left(x-\frac{a_{1}(2 \kappa-\omega)}{1-a_{1} \kappa} t\right)+\xi_{0}\right\}\right]-\lambda}\right) \exp \left(i\left[-\kappa x+\frac{2}{a_{1}} t+\theta_{0}\right]\right),
$$

(1.2) If $\lambda^{2}-4 \mu<0$, and $\mu \neq 0$, it is obtained

$$
\psi_{21,2}(x, t)=\left(A_{0}+\frac{2 A_{1} \mu}{\sqrt{4 \mu-\lambda^{2}} \tan \left[\frac{1}{2} \sqrt{-8 \mu+2 \lambda^{2}}\left\{\left(x-\frac{a_{1}(2 \kappa-\omega)}{1-a_{1} \kappa} t\right)+\xi_{0}\right\}\right]-\lambda}\right) \exp \left(i\left[-\kappa x+\frac{2}{a_{1}} t+\theta_{0}\right]\right),
$$

(1.3) If $\lambda^{2}-4 \mu<0$, and $\mu 0$ and $\lambda \neq 0$, it is gained

$$
\begin{aligned}
\psi_{21,3}(x, t) & =\left(A_{0}+\frac{2 A_{1} \lambda}{\cosh \left[\lambda\left\{\left(x-\frac{a_{1}(2 \kappa-\omega)}{1-a_{1} \kappa} t\right)+\xi_{0}\right\}\right]+\sinh \left[\lambda\left\{\left(x-\frac{a_{1}(2 \kappa-\omega)}{1-a_{1} \kappa} t\right)+\xi_{0}\right\}\right]-1}\right) \\
& \times \exp \left(i\left[-\kappa x+\frac{2}{a_{1}} t+\theta_{0}\right]\right),
\end{aligned}
$$

(1.4) If $\lambda^{2}-4 \mu=0$, and $\mu \neq 0$ and $\lambda \neq 0$, it is gained

$$
\psi_{21,4}(x, t)=\left(A_{0}+\frac{2 A_{1} \lambda^{2}\left[\left\{x-\frac{a_{1}(2 \kappa-\omega)}{1-a_{1} \kappa} t\right\}+\xi_{0}\right]}{-2 \lambda\left[\left\{x-\frac{a_{1}(2 \kappa-\omega)}{1-a_{1} \kappa} t\right\}+\xi_{0}\right]+2}\right) \exp \left(i\left[-\kappa x+\frac{2}{a_{1}} t+\theta_{0}\right]\right),
$$

(1.5) If $\lambda^{2}-4 \mu=0$, and $\mu=0$ and $\lambda=0$, we have

$$
\psi_{21,5}(x, t)=\left(A_{0}+\frac{A_{1}}{\left[x-\frac{a_{1}(2 \kappa-\omega)}{1-a_{1} \kappa} t\right]+\xi_{0}}\right) \exp \left(i\left[-\kappa x+\frac{2}{a_{1}} t+\theta_{0}\right]\right),
$$

From set 2 , it is obtained five general solutions as follows

(2.1) If $\lambda^{2}-4 \mu>0$, and $\mu \neq 0$, it is acquired

$$
\begin{aligned}
\psi_{21,6}(x, t) & =\left(\lambda \sqrt{\frac{2 a_{1}^{3} \kappa^{2}-2 a_{1}^{2} \kappa+2 a_{2} a_{1} \kappa-a_{2}}{a_{3}\left(2 a_{1}^{2} \kappa^{2}-a_{1}^{2} \lambda^{2}+4 a_{1}^{2} \mu-4 a_{1} \kappa+2\right)}}\right. \\
& \left.+\frac{2 A_{1} \mu}{-\sqrt{-4 \mu+\lambda^{2}} \tanh \left[\frac{1}{2} \sqrt{-8 \mu+2 \lambda^{2}}\left\{\left(x-\frac{a_{1}(2 \kappa-\omega)}{1-a_{1} \kappa} t\right)+\xi_{0}\right\}\right]-\lambda}\right) \exp \left(i\left[-\kappa x+\omega t+\theta_{0}\right]\right),
\end{aligned}
$$

(2.2) If $\lambda^{2}-4 \mu<0$, and $\mu \neq 0$, it is obtained 


$$
\begin{aligned}
\psi_{21,7}(x, t) & =\left(\lambda \sqrt{\frac{2 a_{1}^{3} \kappa^{2}-2 a_{1}^{2} \kappa+2 a_{2} a_{1} \kappa-a_{2}}{a_{3}\left(2 a_{1}^{2} \kappa^{2}-a_{1}^{2} \lambda^{2}+4 a_{1}^{2} \mu-4 a_{1} \kappa+2\right)}}\right. \\
& \left.+\frac{2 A_{1} \mu}{\sqrt{4 \mu-\lambda^{2}} \tan \left[\frac{1}{2} \sqrt{-8 \mu+2 \lambda^{2}}\left\{\left(x-\frac{a_{1}(2 \kappa-\omega)}{1-a_{1} \kappa} t\right)+\xi_{0}\right\}\right]-\lambda}\right) \exp \left(i\left[-\kappa x+\omega t+\theta_{0}\right]\right),
\end{aligned}
$$

(2.3) If $\lambda^{2}-4 \mu<0$, and $\mu 0$ and $\lambda \neq 0$, it is gained

$$
\begin{aligned}
\psi_{21,8}(x, t) & =\left(\lambda \sqrt{\frac{2 a_{1}^{3} \kappa^{2}-2 a_{1}^{2} \kappa+2 a_{2} a_{1} \kappa-a_{2}}{a_{3}\left(2 a_{1}^{2} \kappa^{2}-a_{1}^{2} \lambda^{2}+4 a_{1}^{2} \mu-4 a_{1} \kappa+2\right)}}\right. \\
& \left.+\frac{2 A_{1} \lambda}{\cosh \left[\lambda\left\{\left(x-\frac{a_{1}(2 \kappa-\omega)}{1-a_{1} \kappa} t\right)+\xi_{0}\right\}\right]+\sinh \left[\lambda\left\{\left(x-\frac{a_{1}(2 \kappa-\omega)}{1-a_{1} \kappa} t\right)+\xi_{0}\right\}\right]-1}\right) \exp \left(i\left[-\kappa x+\omega t+\theta_{0}\right]\right),
\end{aligned}
$$

(2.4) If $\lambda^{2}-4 \mu=0$, and $\mu \neq 0$ and $\lambda \neq 0$, it is gained

$$
\begin{aligned}
\psi_{21,9}(x, t) & =\left(\lambda \sqrt{\frac{2 a_{1}^{3} \kappa^{2}-2 a_{1}^{2} \kappa+2 a_{2} a_{1} \kappa-a_{2}}{a_{3}\left[2 a_{1}^{2} \kappa^{2}-a_{1}^{2} \lambda^{2}+4 a_{1}^{2} \mu-4 a_{1} \kappa+2\right]}}+\frac{2 A_{1} \lambda^{2}\left[\left\{x-\frac{a_{1}(2 \kappa-\omega)}{1-a_{1} \kappa} t\right\}+\xi_{0}\right]}{-2 \lambda\left[\left\{x-\frac{a_{1}(2 \kappa-\omega)}{1-a_{1} \kappa} t\right\}+\xi_{0}\right]+2}\right) \\
& \times \exp \left(i\left[-\kappa x+\omega t+\theta_{0}\right]\right),
\end{aligned}
$$

(2.5) If $\lambda^{2}-4 \mu=0$, and $\mu=0$ and $\lambda=0$, we have

$$
\begin{aligned}
\psi_{21,10}(x, t) & =\left(\lambda \sqrt{\frac{2 a_{1}^{3} \kappa^{2}-2 a_{1}^{2} \kappa+2 a_{2} a_{1} \kappa-a_{2}}{a_{3}\left(2 a_{1}^{2} \kappa^{2}-a_{1}^{2} \lambda^{2}+4 a_{1}^{2} \mu-4 a_{1} \kappa+2\right)}}+\frac{A_{1}}{\left[x-\frac{a_{1}(2 \kappa-\omega)}{1-a_{1} \kappa} t\right]+\xi_{0}}\right) \\
& \times \exp \left(i\left[-\kappa x+\omega t+\theta_{0}\right]\right),
\end{aligned}
$$

Where $\xi_{0}$ is a constant of integration.

\section{Modulation analysis}

This section of the paper will address the modulation analysis of the unstable solutions. It is well established that bright and dark solitons are stable; thus, it is necessary to verify the regime of the instability of the rest of solutions. On this fact, it will be used the following solutions of Eq. (1).

$$
\psi(x, t)=\left[\sqrt{P_{0}}+A(x, t)\right] e^{i \phi_{N L}}, \quad \phi_{N L}=P_{0} x,
$$

while $P_{0}$ is the incident power. To seek the perturbation, we use Eq. (53) into Eq. (1)

$$
\begin{aligned}
i A_{t}(x, t) & +a_{1} A_{x t}(x, t)+a_{2} A_{x x}+a_{3} P_{0} A(x, t)+a_{3} P_{0}\left(A(x, t)+A^{*}(x, t)\right) \\
& -i \beta P_{0} A_{x}(x, t)-i \beta P_{0}\left(A_{x}(x, t)+A_{x}^{*}(x, t)\right)=0,
\end{aligned}
$$

Hence, Eq. (54) maybe solved in the frequency domain. To do so, it will be considered form of $A(x, t)$ as:

$$
A(x, t)=b_{1} \cdot e^{i(K x-\Omega t)}+b_{2} \cdot e^{-i(K x-\Omega t)} .
$$

Inserting Eq. (55) into Eq. (54) gives the coefficient matrix of $b_{1}$ and $b_{2}$,

$$
\left(\begin{array}{cc}
\Omega+2 a_{3} P_{0}+a_{1} K \Omega-a_{2} K^{2}+2 \beta P_{0} K & \beta P_{0} K+a_{3} P_{0} \\
-\beta P_{0} K+a_{3} P_{0} & a_{1} K \Omega-\Omega-2 \beta P_{0} K+2 a_{3} P_{0}-a_{2} K^{2}
\end{array}\right)\left(\begin{array}{l}
b_{1} \\
b_{2}
\end{array}\right)=\left(\begin{array}{l}
0 \\
0
\end{array}\right) .
$$


The dispersion relation is obtained when a solution of Eq. (56) exists. Spreading out determinant leads to

$$
\begin{aligned}
-4 \Omega \beta P_{0} K & -4 a_{3} P_{0} a_{2} K^{2}-2 a_{1} K^{3} \Omega a_{2}-3 \beta^{2} P_{0}{ }^{2} K^{2}-\Omega^{2}+3 a_{3}{ }^{2} P_{0}{ }^{2}+a_{2}{ }^{2} K^{4} \\
& +4 a_{3} P_{0} a_{1} K \Omega+a_{1}{ }^{2} K^{2} \Omega^{2}=0 .
\end{aligned}
$$

For $a_{1}^{2} K^{2} \neq 1$, it is appear the modulation instability (MI),

$$
\begin{aligned}
& \left(-2 a_{3} P_{0} a_{1} K+2 \beta P_{0} K+a_{1} K^{3} a_{2}\right. \\
& \left.+\sqrt{a_{3}^{2} P_{0}^{2} a_{1}^{2} K^{2}-8 a_{3} P_{0}^{2} a_{1} K^{2} \beta+\beta^{2} P_{0}^{2} K^{2}+4 \beta P_{0} K^{4} a_{1} a_{2}+3 a_{1}^{2} K^{4} \beta^{2} P_{0}^{2}+3 a_{3}^{2} P_{0}^{2}+a_{2}^{2} K^{4}-4 a_{3} P_{0} a_{2} K^{2}}\right)<0
\end{aligned}
$$

and the gain spectrum $G(K)=2 \operatorname{Im}(\Omega)$ and it is revealed

$$
\begin{aligned}
& G(K)=2\left(\frac{1}{a_{1}^{2} K^{2}-1}\right)\left(-2 a_{3} P_{0} a_{1} K+2 \beta P_{0} K+a_{1} K^{3} a_{2}\right. \\
& \left.+\sqrt{a_{3}^{2} P_{0}^{2} a_{1}^{2} K^{2}-8 a_{3} P_{0}^{2} a_{1} K^{2} \beta+\beta^{2} P_{0}^{2} K^{2}+4 \beta P_{0} K^{4} a_{1} a_{2}+3 a_{1}^{2} K^{4} \beta^{2} P_{0}^{2}+3 a_{3}^{2} P_{0}^{2}+a_{2}^{2} K^{4}-4 a_{3} P_{0} a_{2} K^{2}}\right) .
\end{aligned}
$$

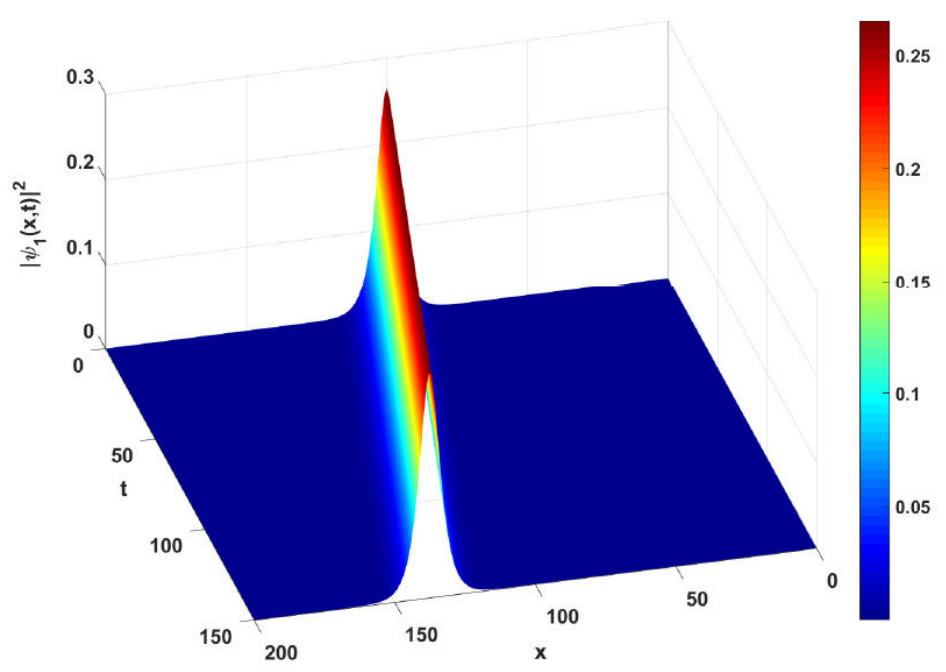

FIGURE 1. Spatiotemporal plot evolution 3-D of the chirp-free bright of $\left|\psi_{1}(x, t)\right|^{2}$ of (12) at $a_{1}=1, a_{3}=1.14, \omega=0.324, \kappa=0.2$, $v=0.25$.
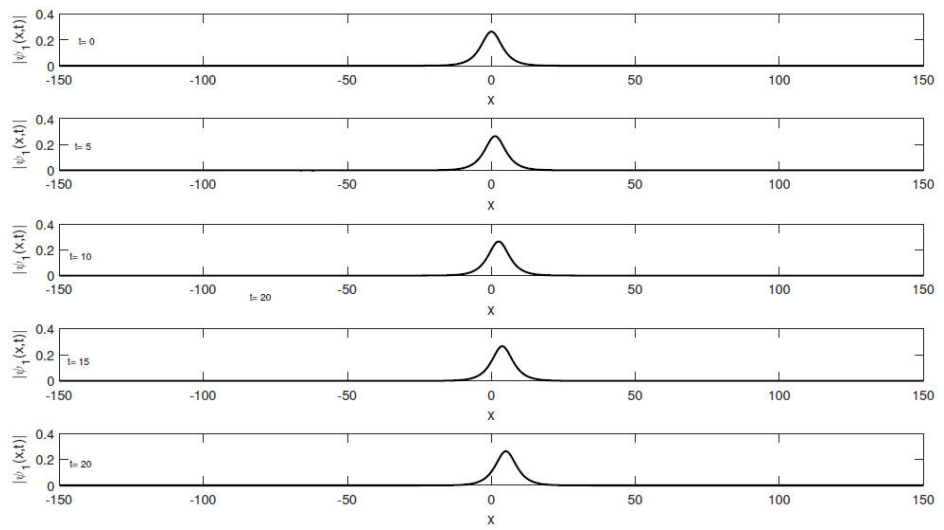

FIGURE 2. Spatiotemporal plot evolution 2-D of the chirp-free bright of $\left|\psi_{1}(x, t)\right|^{2}$ of (12) at $a_{1}=1, a_{3}=1.14, \omega=0.324, \kappa=0.2$, $v=0.25$. 

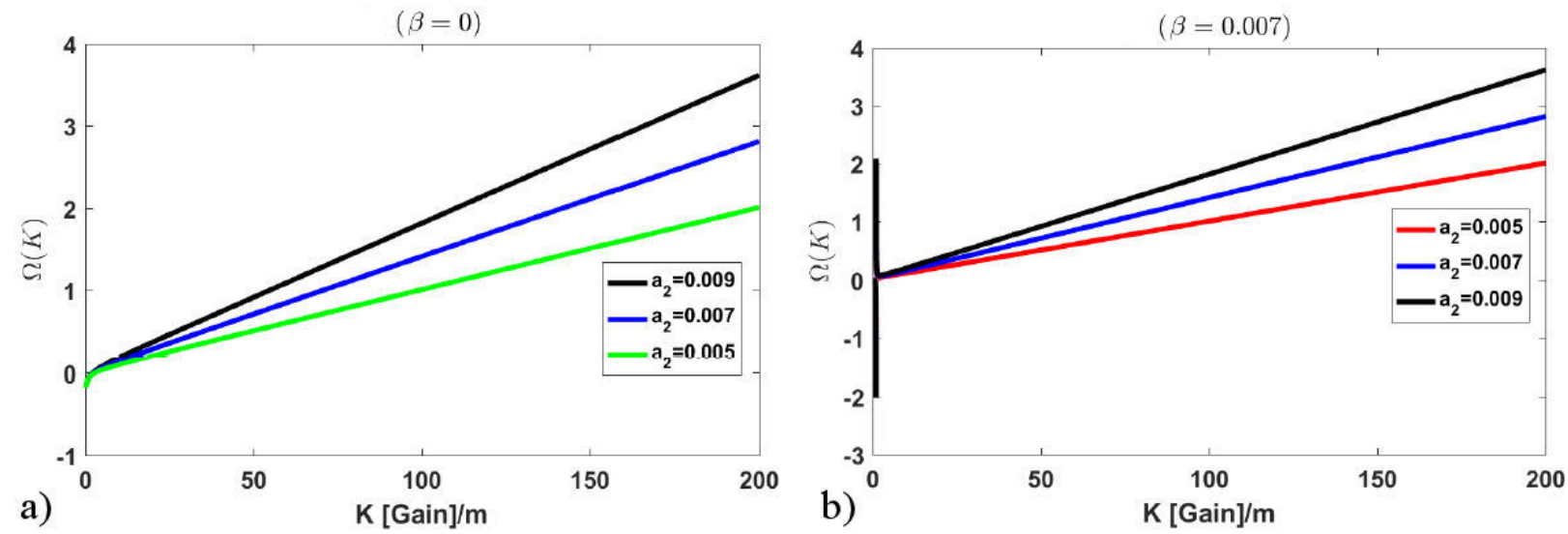

FIGURE 3. The modulation instability gain spectrum a) in the absence of self-steepening $\beta=0$ and $b$ ) with the effect of the self-steepening $\beta=0.007$ at $a_{1}=1, a_{3}=0.007, P_{0}=100$.

Figures 1 and 2 plot the analytical 2D and 3D free-chirp bright solutions for $\left|\psi_{1}\right|$ at at $a_{1}=1, a_{3}=1.14, \omega=0.324$, $\kappa=0.2, v=0.25$. We note that when the self-steepening is absent $(\beta=0)$, it is obtained bright and dark optical solitons with normal-GVD without any deformation of waveform. However, Kerr nonlinearity, STD and low GVD lead to dark solitons to the proposed model of NLSE. In addition, the obtained bright solitons depend on the GVD, STD, and Kerr nonlinearity parameters. Also, it is observed that the (MI) gain spectrum depends on the STD, Kerr nonlinearity and self-steepening parameters; Figures 3a) and 3b) stress the (MI) gain spectrum with the effect of self-steepening. More and more the presence of SPM in the MI gain instability will accentuate the instability zone and the MI gain will grow exponentially. The formation of soliton due the fighting between nonlinear and dispersion terms of the nonlinear Schrödinger equation will be impacted during the propagation in long distance because of the act of SPM and the signal received will be highly perturbed.

\section{Conclusion}

This work studies modulation analysis and optical solitons of PNLSE having STD, perturbation and some nonlinearity terms. We employ the sinh-Gordon technique and $\exp (-\Phi(\xi))$-technique to handle the obtained ordinary differential equation (ODE). Obtained results are bright and dark optical solitons and trigonometric functions. Compare our findings with some previous works $[14,15]$, some additional are obtained by adopting the famous $\exp (-\Phi(\xi))$-expansion method. In addition, the STD and the cubic nonlinearity terms have contributed to generation og bright and dark solitons. Thus, in order to verify the instability regime, we used the modulation instability (MI), to establish the gain spectrum. From there, two areas emerge, where one is stable and the second unstable. The model will take into account modulations of self-phase and cross-phase in a future work.
1. M. A. Ablowitz and P. Clarkson, Solitons, Nonlinear Evolution Equations and Inverse Scattering (Cambridge University Press, Cambridge, 1991), https://doi.org/10.1017/ CB09780511623998.

2. A. Biswas et al., Resonant optical solitons with dual-power law nonlinearity and fractional temporal evolution, Optik $\mathbf{1 6 5}$ (2018) 233, https://doi.org/10.1016/j.ijleo. 2018.03 .123

3. M. Inc et al., New solitary wave solutions for the conformable Klein-Gordon equation with quantic nonlinearity, AIMS Math. 5 (2020) 6972, https://doi.org/10. $3934 /$ math.2020447

4. R. Fedele, H. Schamel, V. I. Karpman, and P. K. Shukla, Envelope solitons of nonlinear Schrödinger equation with an anti-cubic nonlinearity, J. Phys. A 36 (2003) 1169, https: //doi.org/10.1088/0305-4470/36/4/322
5. A. Ali, A. R. Seadawy, and D. Lu, Soliton solutions of the nonlinear Schrödinger equation with the dual power law nonlinearity and resonant nonlinear Schrödinger equation and their modulation instability analysis, Optik 145 (2017) 79, https : //doi.org/10.1016/j.ijleo.2017.07.016

6. E. M. E. Zayed and K. A. E. Alurrfi, New extended auxiliary equation method and its applications to nonlinear Schrödingertype equations, Optik 127 (2016) 9131, https://doi. org/10.1016/j.ijleo.2016.05.100

7. H. Rezazadeh, M. Inc, and D. Baleanu, New Solitary Wave Solutions for Variants of (3+1)-Dimensional Wazwaz-BenjaminBona-Mahony Equations, Front. Phys. 8 (2020) 332, https: //doi.org/10.3389/fphy.2020.00332

8. M. Al Ghabshi, E. V. Krishnan, M. Alquran, and K. Al-Khaled, Jacobi elliptic function solutions of a nonlinear Schrödinger equation in metamaterials, Nonlinear Stud. 24 (2017) 469. 
9. J. Vahidi et al., New solitary wave solutions to the coupled Maccari's system, Results Phys. 21 (2021) 103801, https: //doi.org/10.1016/j.rinp.2020.103801

10. X.-F. Yang, Z.-C. Deng, and Y. Wei, A Riccati-Bernoulli subODE method for nonlinear partial differential equations and its application, Adv. Differ. Equ. 2015 (2015) 117, https: //doi.org/10.1186/s13662-015-0452-4

11. Q. Zhou et al., Exact optical solitons in metamaterials with cubic-quintic nonlinearity and third-order dispersion, Nonlinear Dyn. 80 (2015) 1365, https://doi.org/10.1007/ s11071-015-1948-x.

12. Q. Zhou, M. Mirzazadeh, M. Ekici, and A. Sonmezoglu, Analytical syudy of solitons in non-Kerr nonlinear negative-index materials, Nonlinear Dyn. 86 (2016) 623, https://doi. org/10.1007/s11071-016-2911-1.

13. S. T. R. Rizvi and K. Ali, Jacobian elliptic periodic traveling wave solutions in the negative-index materials, Nonlinear Dyn. 87 (2017) 1967, https://doi.org/10.1007/ s11071-016-3166-6

14. M. M. El-Borai et al., Dark and singular optical solitons with spatio-temporal dispersion using modified simple equation method, Optik 130 (2017) 324, https://doi.org/ $10.1016 / j . i j l e o .2016 .10 .105$

15. A. I. Aliyu, M. Inc, A. Yusuf, and D. Baleanu, Optical solitons and stability analysis with spatio-temporal dispersion in Kerr and quadric-cubic nonlinear media, $O p$ tik 178 (2019) 923, https://doi.org/10.1016/j. ijleo.2018.10.046

16. A. Houwe et al., Complex traveling-wave and solitons solutions to the Klein- Gordon-Zakharov equations, Results Phys. 17 (2020) 103127, https://doi.org/10.1016/j.rinp. 2020.103127
17. A. Houwe, M. Inc, S. Y. Doka, M. A. Akinlar, and D. Baleanu, Chirped solitons in negative index materials generated by Kerr nonlinearity, Results Phys. 17 (2020) 103097, https:// doi.org/10.1016/j.rinp.2020.103097.

18. A. Korkmaz, O. E. Hepson, K. Hosseini, H. Rezazadeh, and M. Eslami, Sine- Gordon expansion method for exact solutions to conformable time fractional equations in RLW-class, J. King Saud Univ. Sci. 32 (2020) 567, https://doi.org/ $10.1016 / j \cdot j k s u s .2018 .08 .013$

19. D. Kumar, K. Hosseini, and F. Samadani, The sine-Gordon expansion method to look for the traveling wave solutions of the Tzitzéica type equations in nonlinear optics, $O p$ tik 149 (2017) 439, https://doi.org/10.1016/j. ijleo.2017.09.066

20. C. Yan, A simple transformation for nonlinear waves, Phys. Lett. A 224 (1996) 77, https://doi.org/10.1016/ S0375-9601(96)00770-0

21. H. Bulut, T. A. Sulaiman, H. M. Baskonus, and A. A. Sadulyak, New solitary and optical wave structures to the (1+1)-dimensional combined $\mathrm{KdV}$-mKdV equation, Optik 135 (2017) 327, https://doi.org/10.1016/j.ijleo. 2017.01 .071

22. K. Khan and M. A. Akbar, Application of $\operatorname{Exp}(-\Phi(\xi)))$ expansion Method to Find the Exact Solutions of Modified Benjamin-Bona-Mahony Equation, World Appl. Sci. J. 24 (2013) 1373.

23. A. Houwe et al., Solitons solutions of nonlinear Schrödinger equation in the left-handed metamaterials by three different techniques, J. Phys. Commun. 3 (2019) 011002, https: // doi.org/10.1088/2399-6528/aaff2c 